Artículos Resultado de la Investigación

\title{
La formación de los estudiantes de derecho, bajo el paradigma de la investigación sociojurídica
}

\section{The training of law students under the paradigmof the research sociolegal}

\section{A formação de estudantes lei sob o paradigma do sociolegais}

\author{
María Teresa Carreño Bustamante ${ }^{1}$ \\ ${ }^{1}$ Universidad de Manizales, Colombia
}

\section{Resumen}

Este artículo aborda las concepciones de participación de los estudiantes de Derecho en Colombia, atendiendo a un supuesto que problematiza la puesta en escena del profesional del derecho una vez egresa del programa, en el que no se evidencia una relación coherente con los postulados de ciudadanía, democracia, valores, civismo que se supone contiene la formación de dicho estudiante, por tener como referente fundamental en su profesionalización la C.N. de 1991.

Palabras clave: ciudadanía, democracia, valores, civismo

\begin{abstract}
Conceptions of the participation of students of law in Colombia, attending a course problematizes staging professional right after she graduated from the program in a consistent relationship to the principles of citizenship, democracy, values are not evidence, citizenship that is supposed to contain the student's education law, having as a fundamental reference in its professionalization CN 1991.
\end{abstract}

Key words: citizenship, democracy, values, civics

\begin{abstract}
Resumo
Concepções da participação de estudantes de direito na Colômbia, assistir a uma problematiza curso de preparo profissional certo depois que ela se formou no programa em um relacionamento consistente com os princípios de cidadania, democracia, os valores não são provas, a cidadania que deveria conter lei de educação do aluno, tendo como referência fundamental na sua profissionalização CN 1991.
\end{abstract}

Palavras-chave: cidadania, democracia, valores, educação cívica

Correspondencia: María Teresa Carreño Bustamante. Universidad de Manizales. revistalogos@policia.edu.co 


\section{Introducción}

El proyecto de investigación del que deviene el presente artículo se ha preguntado por las concepciones de participación de los estudiantes de Derecho en Colombia, atendiendo a un supuesto que problematiza la puesta en escena del profesional del derecho una vez que egresa del programa, en el que no se evidencia una relación coherente con los postulados de ciudadanía, democracia, valores, civismo que se supone contiene la formación de dicho estudiante, por tener como referente fundamental en su profesionalización la Constitución Nacional de 1991.

Uno de los aspectos estudiados son los procesos de formación, atendiendo al mismo supuesto en el sentido de que la teoría en la que se forma a los estudiantes de derecho debería influir en su posterior desempeño profesional, al igual que los procesos que se usan para dicha formación. En este sentido se pone en discusión que la formación a través de procesos investigativos constituye una apuesta importante para alcanzar la formación de un sujeto emancipado, autónomo y reflexivo que es la búsqueda de la mayoría de los programas de Derecho en Colombia y por extensión de los programas de Derecho de otros países latinoamericanos.

\section{Problematización}

¿Cómo formar profesionales del Derecho que respondan individual y colectivamente a las necesidades de su medio? Obligatoriamente es necesario remitirse a la apuesta de formación que cada programa ha concebido y aunque parezca vetusto el tema, se hace necesario reconocer cuál es el objeto de estudio del derecho, o al menos definir cuál es el objeto de formación, o qué postura asumir para realizar dicha formación. Para ampliar esta problematización apelaré a la discusión de trabajo realizada con el grupo de investigación jurídica de la $\mathrm{CUE}^{1}$, con quienes se han puesto de presente algunos avances teóricos del proceso investigativo "Concepciones de participación de los estudiantes de derecho en Colombia", y que contribuyeron a la comprensión del tema. Ampliación de esta problematización puede verse en el proyecto de investigación citado y en el documento de registro calificado del programa de Derecho de la CUE, en el que se tuvo el honor de participar.
El objeto de estudio del Derecho está constituido por los principios teóricos y epistemológicos que lo definen como ciencia, la normatividad que regula las relaciones sociales y los comportamientos que devienen de dichas relaciones; por lo que el objeto de estudio del Derecho tiene una triple dimensión: ciencia, disciplina, profesión.

Desde el punto de vista teórico, el derecho ${ }^{2}$ es uno de los que tiene mayor tradición y desarrollo teórico a lo largo de la historia, dado que fue y ha sido el medio por el cual se regulan las relaciones y los comportamientos de una sociedad y es en este componente -regulación de la vida social- donde cobra sentido la norma y sus diferentes mecanismos de aplicación.

El derecho, ${ }^{3}$ como el medio a través del cual se regula el orden social, contempla concepciones sobre los conocimientos, actitudes y acciones que orientan el quehacer de los sujetos en los diversos ámbitos en los que transcurre su vida. Sin embargo, tales concepciones varían de acuerdo con la época y el período histórico que se viva, pues en la medida en que la sociedad se transforma, también lo hacen la norma y los aspectos legales y jurídicos que la sustentan, de manera tal que se responda permanentemente a los requerimientos sociales.

Así, el derecho es el producto que se genera de la interacción entre los sujetos y de ellos con el Estado, que están mediadas por relaciones políticas, económicas, sociales y por los discursos circulantes de tipo cultural, religioso, político, social, etc. Según palabras de D. Loschak,

(...) la fuerza actuante del derecho no reside solamente en una violencia física extrínseca, ella se organiza en el poder propio del discurso: el derecho es una palabra que se impone como legítima, como verdadera, mucho más allá del círculo restringido de aquellos a los cuales cada una de estas normas, tomada aisladamente, tiene propensión para aplicarse. (Loschak, 1993, p. 34)

En este sentido el derecho es un producto cultural objetivado en las normas que sirven a un modelo de Estado determinado, "el derecho nace de la trascendencia del hombre sobre la realidad social para transformarla, a fin de alcanzar las metas propuestas" (Giraldo, 1999).

El derecho como ciencia, busca inicialmente adherirse al modelo de las ciencias clásicas 
donde los criterios de validez y cientificidad estaban definidos por la capacidad de experimentar y comprobar los enunciados con los cuales daban explicaciones a la realidad. Aunque esta concepción predominó fundamentalmente en las ciencias naturales, dado que en este campo del conocimiento se produjo el desarrollo inicial del método científico, luego se extendió a las ciencias sociales, convirtiéndose en el paradigma dominante; ejemplo de ello para la ciencia jurídica lo constituye la concepción del derecho penal dentro de los "supuestos epistemológicos de las ciencias causales, al pensar que el delito es un comportamiento determinado por factores biológicos"(Lombroso y Garófalo, 1999)(Giraldo, 1999).

Posteriormente la ciencias sociales crearon sus propios criterios de validez a partir de los desarrollos de las teorías contemporáneas sobre la realidad como un constructo social que emerge de las interacciones humanas, las cuales constituyen su objeto de estudio. Con estas teorías se pone en crisis el paradigma positivista del conocimiento y se privilegian las interpretaciones intersubjetivas como nuevo paradigma para comprender los fenómenos de la realidad social. En este sentido el derecho pudo desarrollarse como una ciencia social en el entendido de que ya no tenía la obligación de comprobar sus enunciados, sino crear interpretaciones adecuadas a los contextos sociales y culturales que fueran efectivas para regular las relaciones de las personas.

De este modo, el Derecho como forma del saber cultural, al igual que otras ciencias sociales, tiene fundamentos ontológicos, epistemológicos y deontológicos, a partir de los cuales se puede llegar a premisas distintas sobre lo que es su objeto de estudio y a estrategias diversas acerca de su aplicación.

Recientemente Habermas, en su libro Facticidad y Validez, intenta superar estas discusiones, de orden epistemológico y filosófico, sobre la base de la teoría de la sociedad y de la teoría de la acción comunicativa, a través de "la elaboración de un planteamiento reconstructivo que haga suyas ambas perspectivas: la de una teoría sociológica del derecho y la de una teoría filosófica de la justicia" (2005, p.69), lo que implica entender el derecho como eje organizacional de la sociedad, como instancia procedimental que hace posible la consolidación de sociedades ordenadas y estructuradas, a través de la interrelación de las corrientes del pensamiento tradicionales del Derecho (el lusnaturalismo, el positivismo jurídico y la sociología jurídica).

Como acertadamente concluye Víctor Manuel Rojas Amandi, sobre el derecho y el Estado democrático de derecho en términos de teoría del discurso: "Habermas plantea una teoría contemporánea del derecho que pretende servir de intermediaria entre las teorías del derecho normativas (Kelsen y Hart), del Derecho Natural (Santo Tomas, Dworkin) y sociológicas (Weber y Luhman)"(Rojas, 2004).

Tal y como lo explican Pablo Raúl Bonorino y Jairo Iván Peña Ayazo (2006), existen dos fundamentos en los que se puede apoyar la adopción de una posición positivista respecto de la definición de derecho:

1. El escepticismo ético (positivismo escéptico), que consiste en la creencia de que no existen juicios morales

objetivamente verdaderos, universalmente válidos y eternos (o bien que, en caso de que existieran, no podrían ser conocidos por el hombre mediante el empleo de la razón).

Hans Kelsen se inscribe dentro de este enfoque con su teoría pura del Derecho, con la cual trata de ofrecer una explicación de la ciencia jurídica, eliminado los elementos sociológicos, políticos y morales. La teoría pura del derecho propone dar una respuesta científica a la pregunta "¿Qué es el derecho?" desde un criterio objetivo y neutral. Para lograr esto, es necesario eliminar toda alusión a los aspectos políticos y morales relacionados con la legitimación de los sistemas jurídicos.

1. La ventaja metodológica (positivismo metodológico) implica poder distinguir entre el derecho que es y el derecho que debe ser, con el propósito de permitir la crítica moral de las instituciones vigentes. Dentro de esta perspectiva, Herbert Hart puede ser considerado el representante más importante, pues no intenta responder directamente a la pregunta ¿Qué es el derecho?, sino distinguir diferentes preguntas que comúnmente se 
han planteado quienes han buscado una definición del mismo, como: “¿En qué se diferencia el derecho de las órdenes respaldadas por amenazas, y qué relación tiene con ellas? ¿En qué se diferencia la obligación jurídica de la obligación moral, y qué relación tiene con ella? ¿Qué son las reglas, y en qué medida el derecho es una cuestión de reglas?"

Habermas por su parte, concibe el derecho como "una forma de saber cultural, a la vez que constituye un importante componente del sistema de instituciones sociales. El Derecho es ambas cosas a la vez: sistema de saber y sistema de acción"( 2005, p.145).

Como saber cultural, puede entenderse como " un texto de proposiciones e interpretaciones normativas", como "un sistema de saber dogmáticamente elaborado, es decir, articulado, elevado a nivel científico y entrelazado con una moral regida por principios"(Habermas, 2005, p. 145).

Como sistema de acción, el Derecho puede entenderse como "institución, es decir, como un complejo de regulaciones de la acción" (Habermas, 2005, p.145), que permite la materialización de lo social y lo moral a través de la eficacia práctica que tienen las normas jurídicas (coerción), de la que carecen los principios morales y sociales.

El profesor de la escuela de filosofía de la Universidad Industrial de Santander, Javier Orlando Aguirre Román, en su artículo “La relación lenguaje y derecho: Jürgen Habermas y el debate iusfilosófico", plantea que esta concepción del derecho desde ambas perspectivas, como sistema de saber y sistema de acción, hace del derecho:

El eje central de la vida humana en general y de convivencia social, pues integra lo moral y lo social en un mismo elemento, haciendo que las pretensiones morales adquieran la efectividad y estabilidad que de lo contrario no tendrían. Así el derecho constituye (como derecho positivo) el elemento fundamental y único de garantía de los acuerdos morales y sociales a los que se llegue mediante el diálogo y la acción comunicativa. (Aguirre, 2008, p. 154)

Es aquí donde se evidencia la relación entre la teoría comunicativa y la teoría social habermasiana con su concepción del Derecho, en cuanto son los miembros de la sociedad, los llamados a solucionar los problemas y asuntos sociales a través de la razón argumentativa, que implica un conjunto de condiciones y reglas del acto de habla, que hacen posible una comunicación orientada hacia el entendimiento y el compromiso de los participantes de respetar las consecuencias de sus acciones lingüísticas. Sin embargo, los acuerdos a los que se llegue como resultado de estos debates solo tienen eficacia fáctica cuando se institucionalizan bajo la forma de una norma jurídica.

De todo lo anterior tenemos entonces, que el estudio del Derecho se puede abordar desde dos dimensiones: desde el campo del conocimiento o del saber y desde el campo de la práctica social o de la acción. (Grupo de trabajo CUE, 2011)

Desde el campo del saber, el objeto de estudio del derecho consiste en la descripción, comprensión, análisis, explicación y hermenéutica de las normas jurídicas en su contenido, aplicación, y vigencia. Así, el estudio del Derecho se puede realizar desde diferentes estructuras o categorías que lo organizan lógica y conceptualmente, y lo instituyen como un compendio de conocimientos sistemáticos y articulados, a saber:

- El derecho objetivo (norma agendi) o conjunto de normas, preceptos y reglas de derecho, que gobiernan la vida de los hombres que viven en sociedad; el derecho subjetivo (facultad agendi), que se refiere a las facultades 0 poderes de que son titulares las personas en el ejercicio de sus derechos y que le sirven para satisfacer sus necesidades en los órdenes patrimonial, familiar, social, político, etc. - El derecho sustancial que consagra en abstracto los derechos de que son titulares las personas, como el derecho civil, laboral, mercantil, etc.; el derecho adjetivo 0 procedimental que establece la forma de la actividad jurisdiccional cuya finalidad es la realización de los derechos sustanciales, como por ejemplo, el derecho procesal civil, el derecho procesal laboral, el derecho procesal penal y el derecho procesal administrativo.

- El derecho positivo o escrito, por medio de leyes, decretos, ordenanzas, acuerdos; y el derecho que no estando escrito, hace parte 
integral del ordenamiento jurídico, como es la costumbre, el derecho natural y los principios generales del derecho.

- El derecho público, que se refiere a la organización del Estado y a las relaciones de las personas con este, como el derecho Constitucional, derecho penal, derecho administrativo, derecho tributario, etc; el derecho privado que regula las relaciones entre particulares, para satisfacer sus necesidades de orden jurídico, en aplicación de normas y preceptos de derecho civil, comercial, enmarcadas en relaciones de carácter familiar, provenientes del matrimonio y el parentesco, 0 relaciones con los bienes y a través de los contratos. Estos a su vez, se subdividen en ramas o materias temáticas que se ocupan de objetos de estudio específicos.

Desde el campo de la acción, el derecho se presenta como una realidad compleja que tiene varias dimensiones: por una parte, el conjunto de normas o reglas que regulan la conducta de las personas que viven en sociedad, en un lugar y un tiempo determinado. En este sentido, el derecho se realiza dentro de un contexto social, cultural, temporal e histórico, para darle soluciones jurídicas a los problemas a los cuales se ve enfrentado el hombre por el hecho de vivir en sociedad, y los cuales sirven de fuente para la creación del derecho escrito (derecho positivo); es la adaptación del principio ubi societas ibi ius "donde hay sociedad hay derecho".

Por otra parte, el derecho constituye el medio para asegurar a los integrantes de una nación los valores de la justicia, la libertad, la equidad, la seguridad y la paz.

Asimismo, el derecho interviene en el mundo social desde los diferentes roles que ejercen los individuos llamados a ser operadores jurídicos (legisladores, jueces, fiscales, abogados, operadores burocráticos), sin olvidar aquellos que ejercen las personas que inevitablemente están inmersas en la norma jurídica, ya que toda actividad humana, por regla general, se encuentra regulada por el derecho (ciudadano, profesional, cónyuge, padre, etc.).

El estudio del derecho como campo de conocimiento se ocupa de la descripción, explicación, análisis, comprensión y hermenéutica de la norma jurídica, a través de la docencia y la investigación; lo que permite al futuro profesional el dominio de la fundamentación teórica de la norma jurídica, (competencia cognitiva disciplinar), su interpretación y argumentación (competencia comunicativa) y la actitud crítica y propositiva frente a la misma (competencia investigativa).

Como campo acción, el derecho se ocupa de los problemas sociales con incidencia jurídica, con el propósito de contribuir al desarrollo del Estado Social de Derecho y aportar en la consolidación de una sociedad más segura, justa, equitativa, libre, pacífica y feliz, para lo cual la formación del profesional en derecho enfatiza en la aplicación de los conocimientos para la solución de los conflictos de su entorno (competencia en gestión, administración y resolución del conflicto), con un compromiso ético y social (competencia ética y responsabilidad disciplinar), a través de la práctica, la investigación jurídica y sociojurídica y la proyección social.

${ }^{1}$ Corporación Universitaria Alexander Von Humbolt, de la ciudad de Armenia, Colombia.

\footnotetext{
${ }^{2}$ En la lectura Introducción al análisis del derecho de Carlos Santiago Nino, los siguientes comentarios acerca de la ambigüedad de la palabra derecho: "la palabra derecho es ambigua, y para colmo tiene la peor especie de ambigüedad, que es, no la mera sinonimia accidental (como la de banco), sino la constituida por el hecho de tener varios significados relacionados estrechamente entre sí. Veamos estas tres frases: - el derecho argentino prevé la pena capital- tengo derecho a vestirme como quiera- el derecho es una de las disciplinas teóricas más antiguas - En la primera frase -derecho- hace referencia a lo que, con más precisión, se llama derecho objetivo, o sea un ordenamiento o sistema de normas por ejemplo un conjunto de leyes, decretos, costumbres, sentencias, etc. En la segunda, se usa como derecho subjetivo, como facultad, atribución, permiso, posibilidad, etc. En la tercera se refiere a la investigación, al estudio de la realidad jurídica que tiene como objeto el derecho en los dos sentidos anteriores. En general y para evitar confusiones, se conviene en que derecho a secas denote el ordenamiento y que los demás sentidos sean mencionados con las expresiones derecho subjetivo y ciencia del derecho."
}

\footnotetext{
${ }^{3}$ Afirma, Carlos Santiago Nino: "derecho es una palabra con significado emotivo favorable, nombrar con esta palabra un orden social implica condecorarlo con un rótulo honorífico y reunir alrededor de él las actitudes de adhesión de la gente. Cuando una palabra tiene carga emotiva, ésta perjudica su significado cognoscitivo. Porque la gente extiende o restringe el uso del término para abarcar con él o dejar por fuera de su denotación los fenómenos que aprecia o rechaza, según sea el significado emotivo favorable o desfavorable." En este escrito se hará referencia a la teoría jurídica o ciencia jurídica.
} 


\section{Discusión}

Al respecto, conviene resaltar que en este momento la investigación juega un papel crucial en la formación de los estudiantes de Derecho, en tanto es posible identificar en esta dimensión el impacto que se puede producir en el grupo social. En los currículos de los programas de Derecho en Colombia, esta dimensión ocupa un papel preponderante como función misional, como eje de formación de los estudiantes, como factor de desarrollo de la ciencia del Derecho y como competencia.

Lo anterior tiene relación con las políticas de investigación que han surgido desde hace doce años en el país, cuando empezó el proceso de consolidación de la investigación en Colombia, del que hacen parte los programas de Derecho, y que no proviene de una reflexión de la comunidad académica que le diera vida a una propuesta investigativa, sino de los lineamientos estatales que determinaron la necesidad de hacer investigación en las universidades y se reglamentó su medición a través de diversos software administrados por Colciencias. En la investigación jurídica y sociojurídica, el crecimiento desde el año 2000 a 2010, fecha en que se hizo la última medición, ha sido exponencial; de 14 grupos reconocidos en 2000 se pasó a 267 grupos escalafonados en la

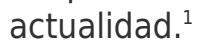

Los productos investigativos rastreados en el GrupLac, están relacionados con las líneas de investigación, de acuerdo con los indicadores de calidad del programa, los productos han generado impacto de visibilidad en tanto la mayoría está publicada en revistas científicas nacionales y algunas internacionales. Las líneas y productos expresan las temáticas que investigan los programas de Derecho. Algunos programas realizan investigación en áreas específicas, por ejemplo en familia.

Otros programas presentan énfasis en el derecho privado, derecho ambiental y derecho público. Los programas con una mayor diversidad temática son los de la Nacional en Bogotá (Pública) que muestra en grupos y líneas una geografía amplia de las diferentes áreas del Derecho, y el programa de Derecho de la Universidad de Medellín, con dos grupos de investigación y una amplia gama de líneas de investigación.

Respecto a la cobertura de la investigación, los grupos tienen un número de investigadores entre uno y 30 , y de estudiantes entre uno y 17 por grupo. El número de estudiantes por programa es muy bajo para el tema de investigación. A pesar de esto, la investigación parece un ejercicio que ha influido el desarrollo de los programas, lo cual se evidencia en los indicadores de Colciencias, con el indicador de artículos en revistas internacionales de niveles altos, lo que pone a una parte de la comunidad académica en diálogo con sus pares internacionales.

A pesar de esto, al revisar los diferentes planes de estudio la investigación no vuelve al aula y, por lo tanto, no impacta a los estudiantes de los programas. Los contenidos se organizan en las asignaturas profesionalizantes y se impactan los estudiantes que participan como auxiliares, asistentes o investigadores, y así se cumple con la cuota de investigación formativa requerida por el MEN, aunque es muy baja la de los estudiantes.

Para Jorge Huergo (2005), la investigación tiene un carácter estratégico-político que impacta la acción de los investigadores respecto de la sociedad que habitan. Su propuesta destaca la constitución de un proyecto de subjetivación o de emergencia del sentido existencial del investigador que se refiere al autoreconocimiento de su carácter de sujeto de la crisis y la transformación, a cambio de pensarse y asumirse meramente como un observador y un intérprete de la realidad, lo que refuerza el divorcio entre teoría y práctica y entre investigación y transformación social. La formación de los estudiantes de Derecho, en este sentido, debiera considerar la investigación como su base pedagógica, pues, vinculada con la formación, la investigación es una praxis ética y ciudadana, lo que permite superar los límites de la imaginación, del pensamiento y del conocimiento:

Son los investigadores quienes determinan los problemas científicos que deben resolverse, definen las relevancias y establecen las metodologías y los ritmos de investigación. Es un conocimiento homogéneo y organizativamente jerárquico en la medida en que los agentes que participan en su producción comparten los mismos objetivos de producción de conocimiento, tienen la misma formación, la misma cultura científica y lo hacen según jerarquías organizacionales bien definidas. (Huergo, 2005, p. 34) 
No puede entonces quedarse la investigación como una mera propuesta normativa para los currículos, pues es necesario volver a ella como mediadora en la formación del sujeto que requiere la sociedad colombiana. No hacerlo es dejarla, como lo plantea Zemelman (2010), como un discurso sin sujeto, donde la persona no importa, porque lo verdaderamente importante son las leyes históricas implacables en su lógica de funcionamiento. Por lo tanto, no contaba, y esto llevó a muchos a construir un discurso brillante, pero sin responsable.

Se requieren diversas apuestas en el proceso de formación, entre ellas la de una identificación del fin para que el que se forma y ello implica un reconocimiento del contexto respecto a la estructura social. Los abogados se forman dentro del marco social, económico y político del país, y esto requiere reconocer que hay una Carta constitucional que guía y regula la interacción, que, aparte de la Constitución, el Estado se ha erigido durante más de quinientos años en formas de organización cultural, diversidad y pluralismo propia de la hibridación de culturas que los constituyen y que estos entrecruzamientos definen diversas formas de organización, de resolución de problemas y de establecimiento de conflictos de toda índole, que evidencian pluralismo, diversidad, multiculturalidad y ello implica diversas visiones del mundo y de comprensión de la realidad.

Lo segundo que se debe tener en cuenta es que la formación debe tocar la conciencia del sujeto con el fin de que se cumpla el interés planteado en las misiones de lograr la transformación del entorno. En este sentido, la acción, en términos de Weber es:

En especial la social y también singularmente la relación social, pueden orientarse, por el lado de sus partícipes, en la representación de la existencia de un orden legítimo. La probabilidad de que esto ocurra de hecho se llama "validez" del orden en cuestión. (Weber, 1944, p. 79)

La acción que se propone en algunos PEI de los programas de Derecho tendría que estar encaminada al reconocimiento de este orden que trata Max Weber respecto a que la validez es el respeto por la regulación de la interacción social. Esto estaría conectado con el para qué de su acción. Es importante entonces, que exista esa iniciativa para que ocurra algo que derive en nuevas construcciones sociales, y esto debe alcanzarse a través del proceso de formación. Es el sentido que Castoriadis sugiere como una paideia democrática que produzca sujetos preparados para la acción que resuene en el conjunto social. En esta forma, se afectarían los currículos de los programas, cuyo propósito es la formación de un sujeto político, lo que expresa la preocupación de los programas por lo político como parte de la formación de los estudiantes, puesto que el Derecho en Colombia como expresión de la sociedad, expresa las condiciones sociales, económicas y políticas del país.

En la política se constituyen sujetos. Algunos son constituidos heterónomamente. Otros se autoconstituyen con mayor grado de autonomía. Incluso en los casos de constitución vertical, siempre hay una dimensión subjetiva que acaba aceptando la interpelación autoritaria y, así, acaba produciendo la relación de subordinación a través de la cual se constituye el sujeto. La política es un campo de fuerzas en el que los sujetos crean instituciones, pero también en el que las pueden destruir, reformar, sustituir. (Tapia, 2008, p. 111)

En esta investigación, el Derecho, promulgado por la CP y desarrollado en las áreas normativas que rigen en Colombia, debe expresarse en el comportamiento social y político de los ciudadanos. La intención de los currículos es la posibilidad de incidir en las prácticas de los profesionales del Derecho. Sin embargo, hay una preocupación por plasmar un discurso convincente pero que no logra realizaciones en la práctica, como señalara Blanco:

Que en tiempos de conflictos entre diferentes concepciones de la sociedad (y por ende de la escuela y el conocimiento que ha de transmitir) el Estado cae con sospechosa frecuencia en la tentación de utilizar la educación como un campo de legitimación (y a los expertos y a los ciudadanos para garantizarla. (Blanco, 1999, p. 195)

Si la política es ese espacio de construcción de las relaciones sociales, dentro de las cuales los sujetos se autorregulan construyen y deconstruyen sus formas de convivencia tal como lo plantea Tapia, "en la política experimentamos tanto el poder de la creación como el de la deconstrucción de lo social y las condiciones que este ha producido para reproducirse, ampliarse, renovarse" (2008, p. 111). El Derecho debe ser parte de esa construcción social en el ejercicio evolutivo de 
acomodación que requiere la sociedad.

La investigación tiene sentido, vista desde la perspectiva de la acción para la formación de los estudiantes de derecho, pues como se afirma en este escrito es un medio para formar la autonomía en el estudiante y lograr en el ejercicio constante de problematización-solución-problematización, la reflexión que se requiere para alcanzar cambios sociales, al mismo tiempo que se generan cambios individuales y se forma el camino para la emancipación individual y colectiva.

${ }^{1}$ Dato tomado de GRUPLACS, Colciencias. 2010.

\section{La formación jurídica se debate entre la CP de 1886 y la de 1991}

La formación de los estudiantes de Derecho en Colombia está influenciada por las dinámicas de educación del siglo XX. Santos y García se refieren así a la formación en educación superior: "fue un conocimiento eminentemente disciplinar, cuya autonomía impuso un proceso de producción relativamente descontextualizado con relación a las necesidades del mundo cotidiano de las sociedades"(2001, p. 34).

Los currículos de los programas de Derecho estudiados están elaborados con los elementos normativos dictados por el Ministerio de Educación. Su organización es teóricamente completa porque desarrolla los objetivos, propósitos y perfiles adecuados a los lineamientos normativos del país. Sin embargo, si se tienen en cuenta las concepciones de participación de los estudiantes encuestados, estos no se forman en coherencia con tales discursos lo que se revela en sus opiniones, sus conceptos y sus prácticas universitarias.

Desde la Constitución de 1991, se viene hablando de una formación que se contextualice con las necesidades sociales, más humana, ubicada en la realidad. Desde el preámbulo de la Carta Fundamental, se ha destacado el valor de la educación al consagrar como elementos que caracterizan el Estado Social de Derecho, la igualdad y el "conocimiento", cuyos bienes consolidan la estructura de un marco jurídico que garantice un orden político, económico y social justo, en función de la prevalencia del interés general.

La declaración de la democracia participativa en Colombia, de acuerdo con el artículo 1 , nos obliga a la configuración de una forma de organización en la que sea posible comprendernos. Son muy diversas las formas en las que el Estado y la sociedad deben reconocer dichas maneras de construirnos, en consideración de la pluralidad, la diferencia y la diversidad que nos caracteriza. Para el caso que nos ocupa, la educación es la fuente de reconstrucción social. Así se plantea en el artículo 67 de la CP:

La educación es un derecho de la persona y un servicio público que tiene una función social; con ella se busca el acceso al conocimiento, a la ciencia, a la técnica, y a los demás bienes y valores de la cultura(...).(CP, 1991, p. 27)

En la ley 30, el artículo 4 dice:

La Educación Superior, sin perjuicio de los fines específicos de cada campo del saber, despertará en los educandos un espíritu reflexivo, orientado al logro de la autonomía personal, en un marco de libertad de pensamiento y de pluralismo ideológico que tenga en cuenta la universalidad de los saberes y la particularidad de las formas culturales existentes en el país. (Ley 30, 1992)

Son muy potentes los intereses que emanan de esta normatividad. El artículo 67 hace referencia a la función social de la educación, pues considera que la formación no tiene una preocupación meramente disciplinar y se espera que tenga un impacto en la sociedad y en las personas, capaz de interiorizar, a través del proceso de formación, los valores de su cultura. Se recaba en el mismo artículo 67“(...)formará al colombiano en el respeto a los derechos humanos, a la paz y a la democracia; y en la práctica del trabajo y la recreación". Por tanto, la pretensión de la Ley no es una formación abstracta sino llevada a la acción.

Ahora bien, la ley 30, al plantear la necesidad de formar un sujeto reflexivo, orientado al logro de la autonomía personal, caracteriza al profesional y al ciudadano. Es preciso reconocer que la CP da las pautas para definir al ciudadano en Colombia, mediante lineamientos normativos. Si Colombia es una democracia participativa y un Estado Social de Derecho, es el ciudadano quien tiene la responsabilidad de desarrollarlo y esto requiere su desarrollo como sujeto participativo. Y solo puede ser participativo quien sea formado en autonomía y reflexividad. Al respecto plantea Kymlicka: 
Autonomía es la capacidad de reflexionar racionalmente sobre nuestros conceptos de vida buena, así como la de tener la facultad potencial de revisarlos. Una persona autónoma es capaz de reflexionar sobre sus fines vigentes y de valorar si esos fines siguen mereciendo su lealtad. (Kymlicka, 2003)

En síntesis, en la intención de la formación en Colombia subyace el interés por la acción, la autonomía y la reflexión. Se esperaría que esta intención propuesta por la Carta, legislada en la ley 30 de 1992, y operacionalizada a través de las diferentes normas que regulan la educación superior en Colombia, se refleje en los PEI de las Instituciones universitarias y los currículos de los programas de Derecho estén organizados de manera que la acción, la autonomía y la reflexión se logren en los estudiantes y en los profesionales del Derecho.

\section{REFERENCIAS BIBLIOGRÁFICAS}

Aguirre Román, J. O. (2008). La relación lenguaje y derecho: Jürgen Habermas y el debate iusfilosófico. Opinión Jurídica,7(13), 139-162.

Blanco, G. (1999). Volver a pensar la educación. En Congreso Internacional de Didáctica, Política, Educación y Sociedad. Madrid: Morata.

Bonorino, P.R., Peña, J. I. (2006). Filosofía del Derecho. Recuperado de http://www.ramajudicial.gov.co/csj/downloads/Us erFiles/File/CSJ/Filosofia\%20del\%20Derecho.pdf

Giraldo, Á. (1999). Metodología y Técnica de la investigación sociojurídica. Bogotá: Legis.
Habermas, J. (1998). Facticidad y Validez. Madrid: Trotta.

Huergo, J. (2005). Los medios y tecnologías Educación. La Plata: Universidad Nacional de La Plata.

Kymlicka, W. (2003). La Política Vernácula, Nacionalismo, multiculturalismo y ciudadanía. Barcelona: Paidós.

Nino, C. S. (2003). Introducción al análisis del derecho. Buenos Aires: Astrea.

Rojas Amandi, V. M. (2004). Facticidad y validez, sobre el derecho y el Estado democrático de derecho en términos de teoría del discurso. Jurídica. Anuario del departamento de derecho de la Universidad Iberoamericana de México, 34, 499-503. Recuperado de http://www.juridicas.unam.mx/publica/librev/rev/j urd/cont/34/pr/pr39.pdf

Santos, B. D., \& García Villegas, M. (2001). El caleidoscopio de las justicias en Colombia. Bogotá: Siglo del Hombre Editores - Universidad de Los Andes.

Tapia, L. (2008). Política Salvaje. La Paz (Bolivia): Muela del diablo editores.

Weber, M. (1944). Economía y Sociedad. México D.F.: Fondo de Cultura Económica.

Zemelman, H. (2010). Pensamiento crítico y neoliberalismo en América Latina. Conferencia Universidad Pedagógica Nacional. Bogotá: Universidad Pedagógica Nacional. 